\title{
Estimation of canopy attributes in beech forests using true colour digital images from a small fixed-wing UAV
}

\author{
Francesco Chianucci ${ }^{a, b, c, *}$, Leonardo Disperati ${ }^{b}$, Donatella Guzzi ${ }^{c}$, Daniele Bianchini ${ }^{\mathrm{d}}$, \\ Vanni Nardino ${ }^{c}$, Cinzia Lastri $^{c}$, Andrea Rindinella ${ }^{\mathrm{b}}$, Piermaria Corona ${ }^{\mathrm{a}}$ \\ a Consiglio per la Ricerca in Agricoltura e l'Analisi dell'Economia Agraria-Forestry Research Centre, viale Santa Margherita 80, 52100 Arezzo, Italy \\ b Università di Siena, Centro di GeoTecnologie, via Vetri Vecchi 34, 52027 San Giovanni Valdarno, Italy \\ ' Consiglio Nazionale delle Ricerche, via Madonna del Piano 10, 50019 Sesto Fiorentino, Italy \\ d Menci Software, Loc. Tregozzano 87, 52100 Arezzo, Italy
}

\section{A R T I C L E I N F O}

\section{Article history:}

Received 1 October 2015

Received in revised form 1 December 2015

Accepted 2 December 2015

\section{Keywords:}

Drone

Sensefly eBee

Leaf area index

Cover photography

Hemispherical photography

Fagus sylvatica

\begin{abstract}
A B S T R A C T
Accurate estimates of forest canopy are essential for the characterization of forest ecosystems. Remotelysensed techniques provide a unique way to obtain estimates over spatially extensive areas, but their application is limited by the spectral and temporal resolution available from these systems, which is often not suited to meet regional or local objectives. The use of unmanned aerial vehicles (UAV) as remote sensing platforms has recently gained increasing attention, but their applications in forestry are still at an experimental stage. In this study we described a methodology to obtain rapid and reliable estimates of forest canopy from a small UAV equipped with a commercial RGB camera. The red, green and blue digital numbers were converted to the green leaf algorithm (GLA) and to the CIE $L^{*} a^{*} b^{*}$ colour space to obtain estimates of canopy cover, foliage clumping and leaf area index $(L)$ from aerial images. Canopy attributes were compared with in situ estimates obtained from two digital canopy photographic techniques (cover and fisheye photography).

The method was tested in beech forests. UAV images accurately quantified canopy cover even in very dense stand conditions, despite a tendency to not detecting small within-crown gaps in aerial images, leading to a measurement of a quantity much closer to crown cover estimated from in situ cover photography. Estimates of $L$ from UAV images significantly agreed with that obtained from fisheye images, but the accuracy of UAV estimates is influenced by the appropriate assumption of leaf angle distribution.

We concluded that true colour UAV images can be effectively used to obtain rapid, cheap and meaningful estimates of forest canopy attributes at medium-large scales. UAV can combine the advantage of high resolution imagery with quick turnaround series, being therefore suitable for routine forest stand monitoring and real-time applications.
\end{abstract}

(C) 2015 Elsevier B.V. All rights reserved.

\section{Introduction}

Accurate estimates of forest canopy are central for a wide range of studies including hydrology, carbon and nutrient cycling, and global change. Leaf area index $(L)$, canopy cover and clumping index are amongst the most widely used canopy attributes. Ground-based methods to estimate these variables include either direct contact (destructive sampling) or indirect optical methods (for a review, see Jonckheere et al., 2004). Generally, both approaches are time

\footnotetext{
* Corresponding author at: Consiglio per la Ricerca in Agricoltura e l'Analisi dell'Economia Agraria-Forestry Research Centre, viale Santa Margherita 80, 52100 Arezzo, Italy. Fax: +39 0575353490 .

E-mail address: fchianucci@gmail.com (F. Chianucci).
}

consuming and unpractical for large forest areas. In addition, these methods are unsuitable for real-time application such as monitoring wildfire spread or plant disease outbreaks (Anderson and Gaston, 2013). Remotely-sensed information offers a unique way to obtain large scale mapping of forest canopy attributes. In particular, several studies indicated that space-borne sensors can be used to obtain spatially extensive information from landscape to the global scale (e.g., Davi et al., 2006; Turner et al., 1999; Hu et al., 2007; Lamonaca et al., 2008; Pellikka et al., 2009; Prospatin and Panferov, 2013). However, the spatial and temporal resolutions of satellite-based data are often not suited to meet regional or local objectives. New satellite sensors have become operational over the past decade, offering data at finer spatial scale and more responsive capabilities. Notwithstanding these improvements, high-cost 